\section{El río Meta en el proceso de fronterización de la frontera colombiana, 1532-2016}

\author{
The Meta River in the Frontier-Making \\ Process of Colombia, 1532-2016
}

\author{
Diana Carolina Ardila-Luna \\ Universidad de los Andes \\ Bogotá, Colombia \\ dc.ardila10@uniandes.edu.co \\ (iD) 0000-0002-6794-8985
}

\section{Información del artículo \\ Recibido: 15 mayo 2020 \\ Revisado: 14 septiembre 2020 \\ Aceptado: 9 febrero 2020}

\section{ISSN 2340-8472 \\ ISSNe 2340-7743 \\ DOI 10.17561/AT.19.5520}

\footnotetext{
CC-BY

(c) Universidad de Jaén (España).

Seminario Permanente Agua, Territorio y Medio Ambiente (CSIC)
}

\section{RESUMEN}

El río Meta es el más importante de los Llanos Orientales colombianos. Ha sido navegado con diferentes fines desde tiempos prehispánicos y conecta a la región con los Andes y el Atlántico. Este artículo tiene como objetivo mostrar la incidencia del río Meta en el proceso de penetración del capitalismo, que contribuyó a creary consolidar la condición de la región como una frontera. Desde la perspectiva de la historia ambiental se revisaron fuentes primarias y secundarias para indagar la incidencia del río en el proceso de "fronterización de la frontera", en la cual ha perdurado la idea de los Llanos como espacio vacío y salvaje. De esta manera, el río ha sido un instrumento utilizado para perpetuar la continua extracción de recursos, vinculada de manera específica a los circuitos del capital y que transformó profundamente sus paisajes.

PALABRAS CLAVE: Historia ambiental de ríos, Río Meta, Frontera

Capitalismo, Orinoquia.

\section{ABSTRACT}

The Meta River is the most important waterway in the Colombian Eastern Plains. It has been navigated for different purposes since pre-Hispanic times and connects the region with the Andes and the Atlantic. This article aims to present the history of the role played by the Meta River as an instrument for the penetration of capitalism that contributed to creating and consolidating the Eastern Plains' status as a frontier. From a perspective of environmental history, a review of primary and secondary sources was carried out to inquire about the impact of the river in the process of frontiermaking, which has generated the idea of the Llanos (Plains) as an empty and wild space. In this way, the river has been an instrument used to perpetuate the continuous extraction of resources that have been specifically linked to the circuits of capital that have profoundly transformed its landscapes.

KEYWORDS: Environmental history of rivers, Meta River, Frontier, Capitalism, Orinoquia. 


\section{Meta River no Processo de Fabricação de Fronteiras da Colômbia, 1532-2016}

\section{SUMÁRIO}

O rio Meta tem sido o rio mais importante da região das planícies colombianas, e é navegado para diferentes fins desde os tempos pré-hispânicos e conecta a região com os Andes e o Atlântico. Este artigo tem como objetivo contar uma parte da história do rio Meta como um instrumento de penetração no capitalismo que contribuiu para criar e consolidar o status da região como fronteira. Do ponto de vista da história ambiental, foi realizada uma revisão de fontes primárias e secundárias para investigar a incidência do rio, ou seja, sua agência, em um processo que acabou na fronteira com a fronteira, perpetuando a ideia dos Llanos como espaços vazios. e selvagem. Dessa forma, o rio tem sido um instrumento utilizado para perpetuar a extração contínua de recursos da região, que estava especificamente ligada aos circuitos da capital e transformava profundamente suas paisagens.

PALAVRAS-CHAVE: História Ambiental dos Rios, Rio Meta, Fronteira,

Capitalismo, Orinoquia.

\section{Le fleuve Meta dans le processus de création de frontières en Colombie, 1532-2016}

\section{RÉSUMÉ}

La rivière Meta a été la rivière la plus importante de la région des plaines orientales colombiennes, elle a été utilisée à différentes fins depuis l'époque préhispanique et relie la région aux Andes et à l'Atlantique. Cet article vise à raconter une partie de l'histoire de la rivière Meta en tant qu'instrument de pénétration du capitalisme qui a contribué à créer et à consolider le statut de frontière de la région. Du point de vue de l'histoire de l'environnement, un examen des sources primaires et secondaires a été effectué pour s'enquérir de l'incidence du fleuve, c'est-àdire de son agence, dans un processus qui a fini par border la frontière, perpétuant l'idée de Los Llanos comme un espace vide. et sauvage. Ainsi, le fleuve a été un instrument utilisé pour perpétuer l'extraction continue des ressources de la région, spécifiquement liée aux circuits du capital et qui a profondément transformé ses paysages.

MOTS CLÉS: Histoire environnementale des rivières, Rivière Meta,

Frontière, Capitalisme, Orinoquia.

\section{Il fiume Meta nel processo di creazione della frontiera della Colombia, 1532-2016}

\section{SOMMARIO}

Il fiume Meta è stato il fiume più importante della regione delle pianure orientali colombiane, è stato navigato per scopi diversi dai tempi pre-ispanici e collega la regione con le Ande e l'Atlantico. Questo articolo mira a raccontare una parte della storia del Meta River come strumento per penetrare il capitalismo che ha contribuito a creare e consolidare lo status della regione come confine. Dal punto di vista della storia ambientale, è stata effettuata una revisione delle fonti primarie e secondarie per indagare l'incidenza del fiume, cioè la sua agenzia, in un processo che è finito al confine con il confine, perpetuando l'idea dei Llanos come spazio vuoto. e selvaggio. In questo modo, il fiume è stato uno strumento utilizzato per perpetuare la continua estrazione delle risorse della regione, che era specificamente legata ai circuiti del capitale e ha profondamente trasformato i suoi paesaggi.

PAROLE CHIAVE: Storia ambientale dei fiumi, Fiume Meta, Frontiera, Capitalismo, Orinoquia. 


\section{Introducción}

Este artículo tiene como objetivo mostrar la incidencia del río Meta en el proceso de penetración del capitalismo, lo que llevó a la consolidación de la "fronterización de la frontera" de los Llanos. Este trabajo fue realizado desde una perspectiva de la historia ambiental -con una mirada de la ecología política y la antropologíacon la intención de analizar desde una perspectiva temporal el lugar del río en el proceso de configuración de la región desde la colonización europea en el siglo XVI. La investigación abordó de manera interrelacionada dos temas importantes para América Latina: por una parte, el estudio de los ríos y, de otra, la frontera.

Respecto al primero, los ríos han sido centrales en la historia de la humanidad y de las sociedades. Gran parte de los grupos humanos han dependido de un río para existir o del manejo de sus aguas para el surgimiento de las civilizaciones. Sin embargo, se le ha prestado poca atención a su importancia como agentes y su capacidad de incidencia en el desarrollo de las diversas sociedades $^{1}$. Los ríos son ecosistemas con límites difusos, como lo define Worster. Un río debe ser entendido como "un río circular" que conecta a las nubes, la lluvia y las corrientes subterráneas como parte del mismo sistema; tienen un ciclo hidrológico particular, una historia geológica y ecológica, entrando en interacción con las sociedades que los perciben, los significan, los usan y los modifican; pueden ser estudiados como ríos de vida, de riqueza, de entretenimiento, de peligro o inspiración; han sido rutas para la colonización, obras de canalización para el comercio y represas para el crecimiento de ciudades o control de inundaciones ${ }^{2}$.

Desde la historia, los ríos han sido ampliamente estudiados como demostraciones de los avances tecnológicos de la sociedad ${ }^{3}$. Sin embargo, para esta investigación me centré en los aportes de historiadores ambientales que entienden los ríos como agentes que inciden sobre las acciones o intenciones humanas. Es el caso de la investigación de White, The Organic Machi$n e^{4}$, quien analiza la transformación del río Columbia para la producción de energía, aprovechando su curso y potencia para hacer posible una forma de vida. Algo similar hace Worster en su libro Rivers of Empire ${ }^{5}$ quien

\footnotetext{
1. Mauch \& Zeller, 2008.

2. Worster, 1994. Arruda, 2006. Coates, 2013. Mauch y Zeller, 2008. White, 1995.

3. Blackbourn, 2008.

4. White, 1995.

5. Worster, 1985.
}

muestra cómo el manejo del agua permite tener un mayor dominio sobre el espacio, situación que lleva a una profunda transformación del paisaje y, asimismo, de la sociedad.

Respecto a la discusión sobre la frontera desde las ciencias sociales ha sido un tema recurrente para el análisis de América Latina. Desde la historia ambiental es presentado como un campo de alto valor para entender los procesos de mercantilización de la naturaleza que están arraigados a la historia del continente ${ }^{6}$. Desde las ciencias sociales ha sido ampliamente discutido el concepto de frontera, el cual no hace alusión a una característica esencial del espacio sino a una asignación histórico-geográfica que es dinámica, resultado de procesos de producción socio-culturales que construyen espacios periféricos a partir de discursos y prácticas ${ }^{7}$. La frontera es definida y delimitada con respecto a un centro y, en este sentido, debe ser entendida en términos de una relación ${ }^{8}$. Asimismo, como nos ha mostrado Harley, las formas de representación del mundo son reflejo de las relaciones de saber-poder que permiten apropiarse del mundo de formas determinadas y producir conocimiento de esto bajo intereses específicos ${ }^{9}$. Y como veremos más adelante, la concepción de frontera responde justamente a unos intereses específicos provenientes de los mercados internacionales, a partir de los cuales se facilitaba la explotación de los recursos de la región, el uso del río como ruta privilegiada y el mantenimiento de la frontera para justificar todas estas acciones.

El interés por el estudio de la frontera se relaciona con la función social de esta en el contexto de la historia del capitalismo. Como afirma Margarita Serje, las fronteras nacen con el capitalismo como parte de las estrategias espaciales y territoriales de este que siempre está en la búsqueda de anexar nuevos espacios para ser apropiados y explotados; son descritos como "desiertos" para crear una imagen de lugares vacíos de historia y de personas y simultáneamente están llenos de riquezas maravillosas, características que son inherentes a la "condición de posibilidad del proyecto colonial e imperial ${ }^{10}$.

Estas dicotomías definen el carácter fronterizo de los Llanos y hacen evidente lo que investigadores han asociado como "al margen del Estado", y lugar de "ex-

\footnotetext{
6. Sedrez, 2002. Gallini, 2009

7. Núñez, Aliste y Bello, 2014

8. Barney, 2009, 152.

9. Harley, 1989.

10. Serje, 2005 y 2017.
} 
cepción". El primero hace referencia a lugares que no están del todo socializados dentro de la ley y que tienen dificultades para ser leídos por el Estado ${ }^{11}$, como lugares que se ven e imaginan como no mapeados ${ }^{12}$. El segundo como un lugar donde el poder soberano y la ley simultáneamente excluyen estos lugares por no hacer parte del orden ${ }^{13}$. Sin embargo, como anota Serje, estos espacios que "están fuera" realmente hacen parte de aquello que los excluye, incluso, le dan sentido a la existencia del orden, la ley y el poder soberano; de tal forma que lo que se establece en realidad es una relación entre la exclusión y la inclusión "mediante las cuales se crean formas particulares de orden social"14.

Para el caso de los Llanos, los investigadores se han referido al concepto de frontera desde diversas perspectivas: como una línea divisoria entre la civilización y la barbarie -al estilo turneriano-, perspectiva compartida por los jesuitas que describieron la región como un espacio desértico y "vacío" de humanidad ${ }^{15}$. También ha sido analizada como una frontera móvil entre indígenas y colonos en una disputa permanente por la tierra como lo describe Gómez en relación a la apropiación de tierras con ganado como estrategia para colonizar la tierra ${ }^{16}$. Rausch define a los Llanos como una frontera permanente, es decir, como un espacio configurado a partir de las relaciones de los Llanos con el centro del país y los procesos de mestizaje de la región que como resultado produjeron particularidades étnicas y culturales, entre ellas los llaneros ${ }^{17}$. Desde los discursos oficiales del siglo XIX, los Llanos fueron concebidos como una frontera agrícola, dada la escasa población y la abundancia de baldío que estaba disponible para la colonización y producción; discursos que en el siglo XXI permanecen ${ }^{18}$. Y una última perspectiva analizada es como área en el margen del Estado, como fuera del orden administrativo, económico y social ${ }^{19}$.

Una aproximación útil para analizar el caso de los Llanos es el concepto de "frontera de recursos", utilizado en la ecología política para referirse a las "nuevas" fronteras que se crean en el mundo después de la II Guerra Mundial y que aparecen como un "descubrimiento" de suministros de nuevas necesidades globales que son accesibles gracias a nuevas tecnologías y organización de la economía glo-

\footnotetext{
11. Das y Poole, 2004.

12. Tsing, 2005.

13. Agamben, 2004

14. Serje, 2013,114

15. González, 2015.

16. Gómez, 1991.

17. Rausch, 1994.

18. Departamento Nacional de Planeación, 2014.

19. Calle, 2016.
}

$\mathrm{bal}^{20}$. Sin embargo, como muestra Majumdar, esta noción no es exclusiva del siglo XXI pues las fronteras de recursos se caracterizan por producir un "peculiar régimen legal y cultural de extracción" -característico de contextos coloniales - como el que describe para la explotación de caucho en la India en el siglo $\mathrm{XIX}^{21}$. Y dicho régimen se caracteriza por un proceso permanente de confinamiento [enclosure] y mercantilización [commodification], propios del capitalismo, que inician con la creación de fronteras en "espacios de la vida social que no han sido colonizados por las relaciones de producción capitalista y sus formas de acción"22. A este proceso, desde la perspectiva de la frontera de recursos, Tsing lo llama "vaciamiento del paisaje", porque implica un cambio de los ecosistemas y las poblaciones en su interior y su distribución ${ }^{23}$.

\section{Área de estudio}

El Meta forma parte de una extensa red de ríos, lagunas, caños, humedales, esteros y morichales ${ }^{24}$ que hacen del agua el elemento principal. El río Meta tiene una extensión de $850 \mathrm{~km}$ y recorre las sabanas tropicales de los Llanos colombianos en sentido este-noroeste hasta desembocar en el río Orinoco. Forma parte de la macrocuenca del Orinoco, que comparten Colombia y Venezuela. En Colombia la Orinoquia corresponde al 33,4 \% del territorio nacional y las sabanas a un $22 \%{ }^{25}$. A nivel administrativo recorre y delimita los departamentos del Meta, Casanare, Vichada y Arauca. Estos departamentos congregan el 3,3\% de la población del país, una de las zonas menos densamente pobladas y, simultáneamente, una de las de mayor diversidad cultural. El $50 \%$ de su población es indígena y vive en resguardos ${ }^{26}$; seguida por la población llanera -campesinos mestizos que poblaron la región antes de la primera mitad del siglo $\mathrm{XX}$ - y los colonos - campesinos migrantes que llegaron después de 1950- ${ }^{27}$.

\footnotetext{
20. Barney, 2009.

21. Majumdar, 2016, 26.

22. Angelis, $2004,72$.

23. Tsing, 2005

24. Los morichales constituyen ecosistemas representativos de la Orinoquía y corresponden a comunidades vegetales en los cuales predomina la palma de moriche (Mauritia flexuosa)

25. Romero-Ruiz et al., 2012.

26. Unidad territorial creada durante la Colonia donde los indígenas vivían agregados. La creación de resguardos respondió a políticas de protección y administración de los indígenas y a procesos de regulación de las relaciones de los colonos españoles con ellos.

27. Sánchez, 2003. Viloria, 2009.
} 
El río Meta ha sido la ruta fluvial más importante de la Orinoquia colombiana tanto por su extensión y caudal, como por su conexión con el río Orinoco y su cercanía a las regiones andina, amazónica y caribeña (Ver Mapa 1). En tiempos prehispánicos, los diferentes pueblos indígenas se movilizaban por una extensa zona de intercambio que comunicaba a la Orinoquia con otras regiones e incluso con el Pacífico, y sus vías principales fueron los ríos ${ }^{28}$. Durante la época colonial, el Meta fue vinculado a las rutas comerciales del Atlántico que lo conectaron con Europa ${ }^{29}$. En los próximos años, se espera que el río forme parte del corredor multimodal que conectará al océano Atlántico con el océano Pacífico en el marco de los proyectos de la Iniciativa para la Infraestructura Regional de Suramérica (IIRSA) ${ }^{30}$.

La región de la Orinoquia, también conocida como Llanos Orientales ${ }^{31}$, incluye una amplia variedad de paisajes que van desde el piedemonte llanero - entre $500 \mathrm{y}$ 700 msnm.- a las sabanas bien drenadas e inundables. Esta extensa área ha sido muy importante para la provisión de materias primas para las regiones cercanas y, desde el siglo XVII, para los mercados internacionales. Quina, sarrapia, plumas, caucho, frutas, yuca, café, carne y cueros fueron importantes durante la colonia y en los últimos años lo han sido los hidrocarburos y los alimentos producto de la agroindustria ${ }^{32}$. Todos estos productos salieron de la región por el río Meta, configurándose una región que -a pesar de su riqueza en recursos- ha permanecido en una condición de frontera a lo largo del tiempo. Los argumentos de esta aparente contradicción se desarrollan en el siguiente apartado.

\section{Metodología}

La perspectiva histórico-ambiental del río Meta se realizó a partir de una investigación de archivos documentales relacionados con la navegabilidad del río Meta, caso del archivo del INVIAS (Bogotá), del de la CORPES en la biblioteca Luis Ángel Arango, así como de la colección de la misma biblioteca y de documentos disponibles en Internet (proyecto IIRSA). Inicialmente, la investigación estaba dirigida a analizar un proyecto concreto: el proyecto de Navegabilidad del río Meta formulado en la

\footnotetext{
28. Tovar, 2010, 13.

29. Rausch, 1994.

30. IIRSA, 2008

31. Nombre dado por los jesuitas para describir la forma plana del terreno y su ubicación hacia el oriente.

32. Gómez, 1991. Romero, 1993. Viloria, 2009.
}

década de 1980. Para la contextualización del proyecto en la región, se consultaron fuentes primarias y secundarias sobre la historia de los Llanos, caso de los cronistas jesuitas, e investigaciones académicas. La revisión de estas fuentes hizo evidente que existieron proyectos de navegabilidad anteriores al de la década de 1980 y que estos compartían importantes similitudes con el objeto de estudio de la investigación. De esta manera, dicha revisión amplió la temporalidad analizada y dio como resultado la lectura que presento en este artículo, la cual inicia en la época colonial y finaliza en 2016, momento hasta el cual hice la revisión de literatura. Se propone el año de 1532 como fecha inicial retomando la fecha registrada en la que los conquistadores europeos llegaron al Meta y realizaron las primeras exploraciones por el río.

\section{Resultados: el Meta en la producción de la frontera}

Desde la perspectiva de la historia ambiental, se presentan los resultados del análisis de tres proyectos de navegabilidad en los cuales el río se constituye como una vía fluvial que ha facilitado la penetración del capitalismo desde el siglo XVII y que a la vez ha contribuido a la construcción, consolidación y permanencia de la frontera en la Orinoquia colombiana, proceso denominado como fronterización de la frontera. Desde esta mirada es posible abordar las discusiones en torno al lugar ocupado por América Latina desde su inserción en la economía-mundo en el siglo XVI, entendido como un proceso de larga duración en el cual la "naturaleza" ha tenido un lugar particular ${ }^{33}$.

El río Meta ha sido navegado a lo largo de toda la historia por diferentes ocupantes: indígenas, conquistadores, llaneros, colonos, comerciantes... Y también por visitantes como viajeros, científicos y gobernantes que admiraron la facilidad de la navegación por el río. Sin embargo, como menciono en la introducción, el interés de este artículo se centra en mostrar la incidencia del río en el proceso de inserción en el capitalismo, el cual ha sido promovido principalmente por agentes externos a la región. De igual modo, fue un proceso que produjo muchos conflictos territoriales y culturales, en particular entre indígenas y colonos, pero no haré énfasis en ellos en tanto excedería el tema y el espacio permitidos.

33. Castro, 2000. 
Mapa 1. Área de estudio

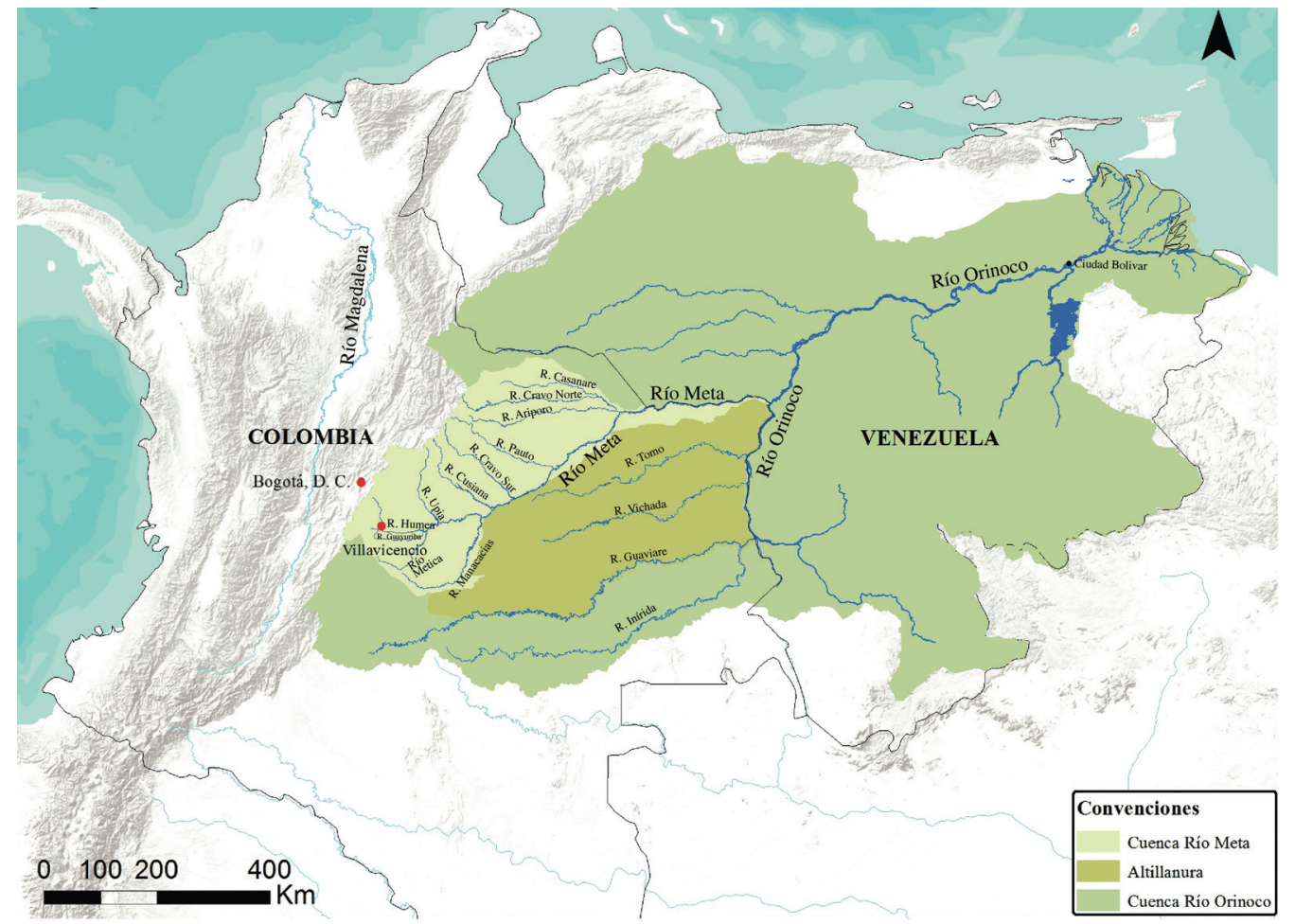

Fuente: Elaboración propia en ArcGIS y finalizado en Illustrator.

\section{La frontera llegó a los Llanos}

En los Llanos colombianos existió una enorme diversidad de grupos indígenas que habitaban la región: achaguas, amorúas, chiricoas, cuivas, curripacos, guahibos, sikuanis, guayaberos, guayupes, macaguane-maciguares, piapocos, piaroas, sálibas, úwas - tunebos- y yaruros ${ }^{34}$. Muchos grupos eran itinerantes y otros sedentarios. Según los investigadores, es poco lo que se sabe de ellos antes de la Conquista. Los estudios arqueológicos han permitido conocer que estos grupos "modificaron su paisaje, construyeron importantes sistemas de camellones aún poco estudiados, lograron generar tierras aptas para la agricultura, tierras pretas con las que aumentaban la productividad natural de la región, y construyeron caminos y calzadas de envergadura" ${ }^{35}$. Los indígenas establecieron amplias redes de intercambio fluviales y terrestres: por el noreste, con los grupos andinos, intercambiaban yopo ${ }^{36}$, algodón y plumas de las tierras bajas por sal, esmeraldas y mantas; por el sur con la Amazonia; también tenían contactos con el mar Caribe e incluso con el Pacífico ${ }^{37}$.

\footnotetext{
34. Romero, 1993.

35. Tovar, 2010, 6 .

36. El yopo (Anadenanthera peregrina) es una sustancia alucinógena utilizada por los indígenas de la Orinoquia y Amazonia colombiana.

37. Langebaek y Morales, 2000. Morey, 1975.
}

Sin embargo, parafraseando a la antropóloga Anna Tsing, "la frontera llegó a los Llanos en el siglo XVI. No siempre había estado ahí" ${ }^{38}$. Con la llegada de los europeos en la primera mitad del siglo XVI, los Llanos se configuraron como un espacio "otro", un espacio "al margen" de la civilización que quedó a la sombra de las montañas de los Andes, donde se ubicó y aún permanece la capital administrativa ${ }^{39}$. De una parte, la cordillera Oriental fue concebida como una "barrera natural" 40 cuyo relieve escarpado hacía difícil el tránsito de personas y productos con las tecnologías europeas y marcaba la separación entre civilizado y salvaje en el marco de una topografía moral de tierras altas/tierras bajas igual a bueno/malo ${ }^{41}$. De otra parte, el clima cálido, los suelos anegados y la población itinerante reforzaron la idea de los Llanos como un espacio límite. De esta manera, la ubicación geográfica y la diversidad cultural se convirtieron en factores determinantes para la construcción de la frontera.

\footnotetext{
38. Tsing, 2005, 30. Ella menciona - para el caso de Kalimantan, en el sudeste asiático- que la región rica en bosques se convirtió en frontera en la segunda mitad del siglo $X X$, una vez sus recursos fueron necesarios para suplir las demandas del mercado internacional.

39. Rausch, 1999.

40. González, 2015.

41. Langebaek y Morales, 2000.
} 
Aun cuando los Llanos se configuraron como una frontera, existía una estrecha relación con los Andes. Una relación desigual en la que la voz dominante de las alturas o tierras altas ha invisibilizado la historia de sus poblaciones y paisajes. Como mencionan Langebaek y Morales: "difícilmente se encuentran en Colombia dos regiones tan cercanas y a la vez tan distantes: cercanas por obvias razones geográficas y distantes porque se les ha estudiado como si se tratara de dos mundos completamente distintos" 42 .

Los primeros conquistadores llegaron a los Llanos siguiendo el curso del río Orinoco y desde los Andes buscando El Dorado ${ }^{43}$. El primer español en llegar fue Diego de Ordaz en 1532, quien "remontó el Orinoco desde el mar (...) hasta los grandes raudales a la altura de Ayacucho, virando luego, guiado por el indígena Tanate, por las bocas del Meta hacia arriba" ${ }^{44}$. Luego pasó Nicolás de Federmán en 1538, proveniente de Coro, hoy Venezuela; Hernán Pérez de Quesada y su hermano Gonzalo Jiménez de Quesada llegaron en 1542 desde el Altiplano Cundiboyacense, hoy Colombia, y luego Antonio de Berrío, quien fue el primero en establecer una ruta entre Bogotá-Río Casanare-Río Meta-Río OrinocoGuyana-Trinidad entre 1584 y $1586^{45}$.

La colonización de los Llanos fue difícil pues las encomiendas y fundaciones de pueblos no funcionaron como en la región andina a causa de la presencia de poblaciones "salvajes" e itinerantes. Como dijimos, el clima cálido, húmedo y las tierras pantanosas de las sabanas inundables contribuyeron a que no fuera un espacio muy apetecido para la agricultura o fundación de pueblos porque se asociaba a la presencia de enfermedades. La Cordillera Oriental se inscribió como una "barrera natural" que dificultaba el tránsito de personas y productos entre las dos regiones. Pero fue, particularmente, la ausencia de oro lo que marcó que la región no fuera prioritaria para la conquista y colonización.

A pesar de las dificultades en la colonización, "la frontera llegó a los Llanos" de las manos y escritos de los misioneros jesuitas, quienes son considerados los verdaderos conquistadores de esta región. Según González en sus descripciones "se encuentran las bases que ayudan a entender la configuración del perfil regional de los Llanos Orientales y la producción so-

\footnotetext{
42. Langebaek y Morales, 2000, 13.

43. Tovar, 2010.

44. Mejía, 2004, 3

45. Mejía, 2004.
}

cial de la diferencias" ${ }^{46}$. Los textos jesuitas resaltaban lo salvaje de las poblaciones, lo "desértico" de las tierras, lo "malsano" del clima y, en claro contraste, lo maravilloso de los ríos. Se referían a los Llanos como "desiertos" para enfatizar su carácter de vacío, en referencia al vacío de humanidad y de cultivos, aunque a la vez estaban maravillados de su riqueza en aguas, pesca y otras formas de alimento ${ }^{47}$. Además, fueron quienes acuñaron el nombre para esta región, pues la describieron como "un inmenso territorio semejante a la mar en calma" 48 .

La permanencia de esta idea de frontera ha sido fundamental en la construcción de las relaciones entre los Andes y los Llanos desde la Colonia hasta la actualidad. En esta aparente contradicción entre lo vacío y la riqueza de recursos se configura la base de las estrategias diseñadas desde el centro del país para integrar la región a los mercados andinos y mundiales. De esta manera, el ingreso de los Llanos a la economía-mundo se da a través de la explotación de recursos de alta demanda y el río fue central en la materialización de las mencionadas estrategias. A continuación, se presentan los tres grandes proyectos estudiados en torno al río Meta los cuales han sido la base fundamental para la consolidación de un proceso de fronterización de la frontera.

\section{El Meta en el proyecto de expansión de los jesuitas}

Frente al problema de la colonización de las tierras de los Llanos, la Corona española buscó implementar otras estrategias para ampliar la frontera colonial e integrar los nuevos territorios.

Con el objetivo de hacer presencia en la región y encargarse de la vida material y espiritual de los indígenas ${ }^{49}$, la Corona entregó mercedes de tierras a diferentes comunidades religiosas para que se instalaran en dichos territorios, evangelizaran y civilizaran a los indígenas, lo que se conoce como "reducción de indios" 50 . La orden de mayor presencia en los Llanos fue la Compañía de Jesús, quienes hicieron su primera entrada en 1625 hasta que les retiraron sus facultades en 1628. Regresaron en 1659 permaneciendo hasta 1767, año de su expulsión ${ }^{51}$.

\footnotetext{
46. González, 2015, 17.

47. González, 2015, 29

48. Rivero, 1956, 1.

49. Cassani, 1741.

50. Colmenares, 1984.

51. Gómez, 1991.
} 
Los religiosos llegaron a las orillas del Meta provenientes de los páramos andinos y del Orinoco, desde el Atlántico. Los jesuitas entendieron que los ríos eran estratégicos para civilizar y evangelizar a los indígenas, pues según las censos del siglo XVI cerca del setenta por ciento de la población vivía próxima a los ríos ${ }^{52}$. El Meta y sus riberas se convirtieron en espacios privilegiados para asentarse. Los ríos proveían un buen lugar de vivienda, agua, alimentos y espacios cultivables. Por ejemplo, Rivero resaltó: "no son estériles los ríos, pues además de los muchos peces, aves y animales que viven a expensas suyas, se crían muchos palmares de diversas especies y varios árboles frutales" ${ }^{53}$. También escribió sobre la abundancia de fuentes de agua: "la distancia que hay de ríos á ríos y de quebradas á quebradas es tan proporcionada, que á dos ó tres leguas de camino se suceden las unas á las otras" ${ }^{24}$. Asimismo, los ríos eran importantes rutas de comunicación con los Andes y con el Atlántico. Cassani menciona que la navegación siempre fue muy cómoda, salvo cuando el río crecía ${ }^{55}$. Para ellos fue importante mantener un contacto entre el Meta y el Orinoco, que proporcionaba una salida al Atlántico y a través de este, incluso de contrabando, lograron importar de Europa herramientas y otros objetos necesarios ${ }^{56}$.

A lo largo del siglo XVII y XVIII las diferentes órdenes religiosas crearon sus misiones en torno a los ríos (Ver Mapa 2). La estrategia expansiva de los jesuitas se hizo siguiendo el curso del río Meta, el Orinoco y sus afluentes. Para garantizar su permanencia en los Llanos, los jesuitas crearon un complejo económico-administrativo constituido por una red de haciendas, hatos ${ }^{57}$, pueblos y misiones que estaban interconectados a través de la red hídrica y así se lograba el control de todo el territorio, además de un control del trabajo, de lo producido, consumido y ganancias obtenidas en cada uno de los espacios $^{58}$. Los hatos, pueblos y misiones dependían administrativa y económicamente de las haciendas ubicadas entre el piedemonte y el Meta. Caribabare fue la más importante y con el tiempo fue el centro administrativo de todas las haciendas. A su vez, todas las instancias dependían del Colegio Máximo de Santa Fe, donde estaba La Tienda, donde se comercializaban los productos de los Llanos; así, lograron establecer una amplia red comer-

\footnotetext{
52. Tovar, 2010.

53. Rivero, 1956, 4.

54. Rivero, 1956, 33

55. Cassani, 1741.

56. Hernández, 2009.

57. El hato es el conjunto de cabezas de ganado o la hacienda de campo destinada a la cría de estos animales.

58. Rueda, 1989, 12.
}

Mapa 2. Idea del curso del río Meta

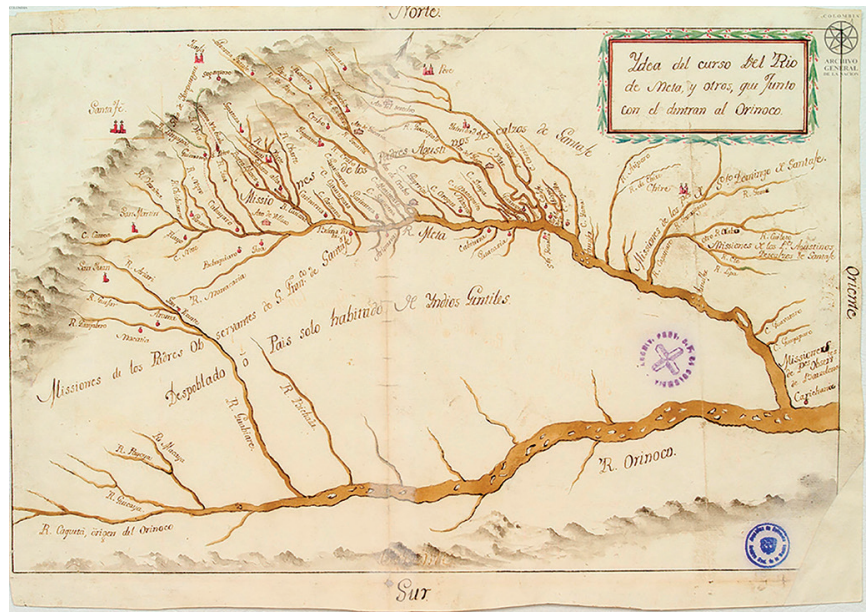

Fuente: Archivo General de la Nación (Colombia). Mapoteca, 4, 680A. Sin fecha.

cial que aseguraba la venta y consumo de sus productos y apropiarse de extensas áreas de la región ${ }^{59}$.

El proceso de evangelización no fue el único objetivo de los jesuitas con los indígenas, también se dio el aprendizaje de una nueva forma de vida basada en el trabajo ${ }^{60}$. El día a día en las misiones se repartía trabajando en las vegas ${ }^{61}$ donde se sembró yuca, maíz y frutas europeas necesarias para la alimentación, con actividades en el área de sabana relacionadas con el ganado, con la pesca, la caza y las labores en la iglesia, entre otras ${ }^{62}$. La fuerza laboral estaba constituida por indígenas, personal concertado y esclavos africanos a los que se les pagaba principalmente en especie dado que circulaba poco dinero en la región. Así avanzó la expropiación de recursos comunes y de territorios a los indígenas, su privatización y con la exportación de los productos - el ganado tenía como destino poblados andinos- se lograba afianzar el avance de la lógica del capital y su acumulación.

De esta manera, los jesuitas hicieron uso de su concepto de frontera para colonizar el territorio en beneficio propio: al ser desiertos y estar poblados por salvajes, los misioneros tuvieron libertad de organizar el territorio, la vegetación y sus poblaciones como más les convenía. Los ríos fueron muy importantes para la creación de la red de las diferentes fundaciones realizadas y, de esta manera, la frontera no fue solo una idea, sino que los jesuitas lograron materializarla en un nuevo paisaje donde predominaba el ganado y la vida urbana que transformaron profundamente la vida cotidiana de los indígenas.

\footnotetext{
59. Rueda, 1989.

60. Colmenares, 1984

61. Espacios cercanos a la ribera de los ríos y caños que cuentan con suelos aptos para la agricultura.

62. Rivero, 1956. Cassani, 1741. Gumilla, 1944.
} 


\section{El Meta y la frontera de la Nueva República}

En 1819 el país se independizó de España y los Llanos formaron parte de la frontera de la Nueva República. Para el siglo XIX se mantenían vigentes los conceptos de los jesuitas sobre la región: desierto, salvajes e importantes recursos. En este contexto, la región ahora era vista desde una perspectiva empresarial que la concebía como el "futuro de Colombia", como una despensa que alimentaría a Bogotá y al mundo ${ }^{63}$. La intención del gobierno central para lograr incrementar la productividad de la región se dirigió a fomentar y auspiciar, con garantías y beneficios, la colonización e iniciativas privadas. Con el objetivo de satisfacer la demanda de productos del exterior ${ }^{64}$ se reanudaron las misiones, se introdujo la navegación a vapor por el río Meta, se construyeron caminos, se fomentó la colonización y la producción de cultivos y materias primas de alta demanda ${ }^{65}$.

Sin embargo, la situación de la región en la primera mitad del siglo XIX no era muy alentadora. Después de la expulsión de los jesuitas en 1767 y las guerras de independencia entre 1810 y 1819, hubo una importante baja demográfica que desestructuró las formaciones territoriales que habían logrado consolidarse durante la colonia, situación que desarticuló aún más a los Llanos con el centro administrativo ${ }^{66}$. Para este momento los Llanos no contaban con carreteras importantes que los comunicara con otras regiones del país, los caminos eran de herradura y seguían el curso trazado por los indígenas en tiempos prehispánicos ${ }^{67}$. Hubo interés en abrir caminos, pero mientras estos proyectos se llevaban a cabo, el río Meta volvió a ser el protagonista para la materialización del proyecto agroexportador.

En la segunda mitad del siglo XIX llegaron nuevas oleadas de colonos provenientes de los Andes y de Venezuela, así como comerciantes extranjeros que ocuparon tierras y las hicieron producir ${ }^{68}$. Las poblaciones indígenas nuevamente vieron sus territorios ocupados acrecentándose los conflictos con los llaneros. Estos nuevos colonos pudieron comercializar sus productos de manera más fácil gracias a la introducción de la navegación a vapor en $1849^{69}$.

\footnotetext{
63. Rausch, 1999, 87.

64. Rausch, 1999.

65. Rausch, 1999, 15.

66. Barona, Gómez y Domínguez, 1998.

67. Langebaek y Morales, 2000.

68. Cobos, 1966

69. Silva, 2009.
}

La navegación comercial promovida por los gobiernos liberales empezó a rendir frutos a finales del siglo XIX. La navegación a vapor fue importante para incrementar el volumen de carga y reducir el tiempo de viaje entre Bogotá y el Atlántico ${ }^{70}$. Antes de los vapores, el recorrido podía tomar cerca de ochenta días, con los vapores tomaba entre cinco y quince días ${ }^{71}$. La navegación a vapor por el Meta, entre 1850 y 1880, estuvo a cargo de compañías venezolanas y algunas colombianas, pero no lograron hacerlo de manera regular a causa de las continuas guerras civiles y los problemas fronterizos con Venezuela ${ }^{72}$. Adicionalmente, los comerciantes de Cartagena se empeñaron en impedir el despliegue de la navegación por el Meta al poner en peligro sus intereses económicos ${ }^{73}$.

El panorama cambió cuando en 1880 los gobiernos de Venezuela y de Colombia otorgaron al francés José Bonnet una concesión especial para la libre navegación y transporte de mercancías en el Meta y Orinoco. Bonnet fue el primero en establecer un comercio regular en la región ${ }^{74}$. El comerciante francés se comprometió a crear puertos y bodegas que facilitaran el transporte de las mercancías ${ }^{75}$. En 1899 logró un nuevo contrato en el que se le concedió un subsidio de tres mil pesos por cada viaje redondo efectivo y se le asignaron 30.000 ha de terrenos baldíos sobre el eje del Meta para que cultivara café, cacao y otros productos exportables ${ }^{76}$. La navegación y el comercio fueron constantes hasta el inicio de la Guerra de los Mil Días en 1899. Durante los enfrentamientos, los combatientes tomaron uno de los barcos de Bonnet y lo destrozaron, empezando así el camino a la quiebra de este empresario y la decadencia de la navegación comercial $^{77}$. La navegación mantuvo alguna regularidad gracias a los incentivos que dio el gobierno, pero en 1930 fue suspendida casi definitivamente como resultado de la crisis económica mundial de $1929^{78}$.

El incremento de la exportación e importación hizo necesario instalar aduanas y otros organismos de control, así como consulados de varios países europeos en Orocué, un pequeño puerto ubicado en la parte media del Meta, que para ese momento se convirtió en el puerto más importante de la región ${ }^{79}$. Asimismo, este

\footnotetext{
70. Franco,1997.

71. Silva, 2009.

72. Rausch, 1999.

73. Bonnet, 1884.

74. Junguito, 2011, 3.

75. Rausch, 1994.

76. Bonnet, 1884.

77. Franco, 1997.

78. Silva, 2009.

79. Franco, 1997.
} 
auge incentivó la migración de colonos hacia las sabanas, lo cual facilitó la apropiación de extensas áreas de tierras o las recibieron como pago por su prestación de servicios durante las diversas guerras ocurridas desde la Independencia ${ }^{80}$. Surgen así los grandes terratenientes de los Llanos ${ }^{81}$. Otros llaneros tomaron posesión de terrenos más pequeños para cultivos y ganado $^{82}$. Unos y otros despojaron a los indígenas de sus territorios, situación que incrementó los conflictos y la violencia en la región y consolidó las economías extractivas que arrasaron con las semillas de sarrapia, quina, pieles de animales y garzas, entre muchos otros productos $^{83}$.

Nuevamente el Meta funcionó como una vía para la penetración del capitalismo y reforzó la condición fronteriza de la región al incrementar las actividades extractivas y facilitar su salida hacia los mercados internacionales. Como sucedió con los jesuitas, este auge se dio cuando el gobierno dejó en manos privadas el destino de la región. De esta manera, grandes cantidades de capital circularon por la región, pero no retornaron.

\section{El Meta y la agroindustria}

Una vez finalizado el auge agroexportador, la navegación comercial del río Meta solo se reactivó en la década de 1940 y a escala nacional. Esta actividad, que continúa hasta la actualidad, se ha centrado en comerciar ganado, plátano y yuca hacia los Andes y el transporte de maquinaria, materiales de construcción y otros víveres hacia los Llanos. Esto fue posible gracias a que el Estado en 1944 creó la Compañía Nacional de Navegación (NAVENAL) con el objetivo de comunicar las vías fluviales del país con el Caribe, Estados Unidos y Europa. Sin embargo, los continuos problemas fronterizos con Venezuela mantuvieron la navegación restringida al territorio nacional. La navegación de NAVENAL en el Meta se realizó desde 1948 principalmente con el transporte de insumos para el ganado y los cultivos. Finalmente, $\mathrm{y}$ al parecer por problemas administrativos, la empresa fue liquidada en $1984^{84}$.

Hacia finales del siglo XX resurgió el interés por los Llanos en un contexto diferente. En la década de 1970 se

\footnotetext{
80. Franco, 1997.

81. Gómez, 1991.

82. Sánchez, 2003.

83. Rausch, 1994.

84. Melendro, 1978.
}

habían hallado importantes yacimientos de petróleo y la investigación científica daba resultados sobre la producción a escala industrial en los suelos de sabana de la altillanura ${ }^{85}$. La relevancia de estos hallazgos representó una posibilidad real de transformar las extensas áreas de sabanas -ocupadas principalmente con ganado- en áreas de cultivo que producirían comida y energía para un mundo cuya población aumentaba y que demandaba mayor cantidad de productos. De esta manera, las sabanas de los Llanos son presentadas al mundo como una de las últimas fronteras agrícolas del planeta. Para $2003 \mathrm{el}$ expresidente Uribe se refiere a la región de la siguiente manera:

\begin{abstract}
"Pero, miren: le veo a esa Orinoquía [sic] un futuro ya inmediato en el agro. En esos 600.000 kilómetros diríamos que hay 350.000 de selva y 250.000 de sabanas. ¿Ustedes saben lo importante que es para el mundo agropecuario tener allí 250.000 kilómetros planos, sin piedra, listicos para cultivar y sin el obstáculo ecológico de que hay que llegar con el hacha?"86.
\end{abstract}

El gobierno siguiente de Juan Manuel Santos expresó ideas similares sobre la Orinoquia en su visita presidencial a Orocué para la sanción de una nueva ley de tierras ${ }^{87}$ :

“El mundo está cada vez más preocupado porque el mundo está creciendo y el mundo necesita alimentarse... Y hay solamente unos pocos países donde hay un verdadero potencial para aumentar esa producción de alimentos, 7, 8 países, uno de ellos es Colombia y dentro de Colombia es toda esta zona, toda la altillanura, que la podemos convertir en una despensa para el mundo entero, en una fuente de prosperidad" $"$.

Para el siglo XXI, las sabanas de la Orinoquia adquieren una nueva significación que complementa la anterior. Dentro de las dinámicas y discusiones ambientales del nuevo milenio, el cambio climático se vuelve un tema central. En esta línea, durante el segundo gobierno de Álvaro Uribe se creó el programa llamado "El Renacimiento de la Orinoquia" que dentro de su nueva propuesta incluía la mitigación del cambio climático.

\footnotetext{
85. Lugari, 1986. García, 2009. Rausch, 2009. La sabanas de altillanura están ubicadas en el costado sur del río Meta.

86. Durán, 2012, 202.

87. Ley ZIDRES. Las Zonas de Interés de Desarrollo Rural, Económico y Social (ZIDRES) son territorios especiales, aptos para la agricultura, la ganadería, la pesca o los desarrollos forestales, pero alejados de los centros urbanos, con baja densidad de población y limitada infraestructura.

88. Santos, 2016.
} 
El programa fue concebido como "proyecto de recuperación de bosque tropical húmedo del mundo [a partir de una] reconversión agroambiental de las sabanas que contribuya al rescate de la atmósfera (...); es una oportunidad única de desarrollo rural sistémico sostenible, que Colombia le ofrece al mundo y a su propia población, con el objeto de contribuir de manera sustancial a la estabilización del clima global" ${ }^{89}$. Estas afirmaciones muestran cómo la noción de frontera como espacio vacío, despoblado y salvaje, pero con muchos recursos sigue vigente después de 400 años.

Con el objetivo de hacer las sabanas productivas, uno de los proyectos propuestos por el gobierno colombiano para esta época fue recuperar la navegabilidad del río Meta en 1987. Este proyecto era estratégico para ofrecer una salida a los productos de la región hacia el comercio nacional e internacional ${ }^{90}$. Posteriormente, en el año 2000, una vez los países del Pacífico se convirtieron en importantes socios comerciales del país, se firmó la Iniciativa IIRSA ${ }^{91}$ en la que se propusieron construir un corredor multimodal entre el Pacífico, de tal forma que los productos de los Llanos llegarían a cualquier lugar del mundo ${ }^{92}$. Este tipo de conectividad solo sería posible a través del río: las carreteras existentes en la región aún son muy pocas y no se diseñaron pensando en este tipo de comercio, así que el río Meta sigue siendo la ruta privilegiada para la vinculación de la región con el comercio exterior.

El proyecto en la actualidad ha hecho pocos avances en la adecuación del río y la construcción de infraestructura para cargar y descargar productos. Hasta la segunda década del siglo XXI se entregaron obras como el puerto de La Banqueta en la parte alta del río y una rampa en el río Manacacías, tributario del Meta. Y otras dos obras están aún sin finalizar. Sin embargo, con la promesa de realización del proyecto se han producido nuevas olas de colonización, esta vez por parte de grandes empresas que tienen la tecnología y el capital necesarios para modificar los suelos de las sabanas y hacerlos productivos a escala agroindustrial. De esta manera, las empresas están imponiendo una nueva territorialidad relacionada con la fase más reciente del capitalismo, el llamado neoliberalismo, en la cual el mercado se sitúa como el regulador social central. En esta nueva iniciativa, el gobierno una vez más ha delegado en manos privadas la intención de superar la con-

\footnotetext{
89. Ministerio de Agricultura y Desarrollo Rural, 2004, 10-11.

90. Pacheco, 1987.

91. Iniciativa para la Infraestructura Regional de Suramérica.

92. IIRSA, 2008
}

dición fronteriza de la región, incluso contemplando la entrega del río en concesión ${ }^{93}$. El resultado ha sido un impulso a las economías extractivas que obtienen grandes ganancias con los recursos de la región, la cuales no retornan a esta y una profunda transformación de las sabanas, las cuales pasaron de ser pastizales a lo que ahora "parecen" bosques.

\section{Conclusión: la "fronterización" de la frontera}

La persistencia de la condición de frontera en la región de la Orinoquia colombiana sigue siendo una pregunta importante para la investigación social. La mirada histórico-ambiental centrada en el río como vía fluvial privilegiada permite ver cómo las características del río -su ubicación y su aptitud navegable - facilitaron la articulación particular de la región en el contexto amplio de la economía-mundo. La navegación del Meta desde la época colonial y la relación establecida con los Andes contribuyeron a crear y consolidar la condición fronteriza como un espacio "al margen" del Estado. Condición que ha logrado permanecer en el tiempo con las mismas ideas y básicamente las mismas estrategias.

La situación fronteriza de los Llanos no se debe a un tema cultural ni político, es decir, no ha sido un problema de sus habitantes o del gobierno sobre la región. Por el contrario, esta situación está fuertemente relacionada con aspectos económicos $\mathrm{y}$, particularmente, con el lugar que la región ocupa dentro de los circuitos mercantiles mundiales. El análisis de los tres proyectos muestra que los Llanos, más que una frontera que divide lo salvaje de lo civilizado o espacio en el margen, han sido construidos como una "frontera de recursos" y el río Meta ha sido el motor de este proceso al haber transportado por sus aguas las diversas materias primas que llegaron a Europa y por las que ingresaron nuevas tecnologías y especies vegetales y animales que transformaron los paisajes. Una frontera abierta hacia el exterior y continuamente intervenida por agentes externos con intereses particulares. Otra característica de esta frontera, como describe Tsing para el caso del sudeste asiático, es el "vaciamiento del paisaje" como una acción constante que tiene como único objetivo extraer recursos para el comercio externo ${ }^{94}$. Asimismo, como menciona Majumdar, este tipo de fronteras

\footnotetext{
93. CyM. Cal Mayor y Asociados, 1999.

94. Tsing, 2005.
} 
se realiza bajo un régimen de explotación, con un régimen legal y cultural particular ${ }^{95}$. En el caso de los Llanos, los proyectos permiten ver que su integración a la economía-mundo fue reiteradamente delegada a las manos privadas, con permisos y legislación excepcional (las misiones en vez de las encomiendas, la licencia de Bonnet, la ley Zidres, entre otras) emitida por los entes gubernamentales.

Es importante resaltar que en los tres momentos abordados -el colonial a través de los jesuitas, el del siglo XIX con el auge agroexportador y el del siglo XX con el crecimiento agroindustrial- se explotaron los recursos y se transportaron hacia los mercados internacionales por el río Meta y esto ha hecho que el río se convierta en un instrumento para fronterizar la frontera. Es decir, la navegación del Meta ha sido utilizada para extraer los recursos, llevarlos a los mercados e importar bienes que no se quedan en la región; todo este ir y venir de productos en ningún momento pretende devolver algo a la región o a sus poblaciones. Mantener en el tiempo la concepción de la frontera, representar a los Llanos como un espacio "al margen" o como un espacio "vacío", ha hecho parte de la estrategia para consolidar y perpetuar un tipo de relación entre la sociedad, el río y los ecosistemas y sus poblaciones.

Fronterizar la frontera también ha permitido eludir explicaciones por los desastres ecológicos y genocidios en la región a causa de ocupaciones de tierras mediadas por la violencia y amparadas por el estado. Asimismo, para justificar intervenciones que se enfocan en lo económico, para pagar favores políticos, para recrear imaginarios de una región inexplorada y salvaje, para invisibilizar sus aportes al país y al mundo desde lo económico y lo cultural. No es nuevo en los Llanos que los recursos sean más importantes que las personas.

\section{BIBLIOGRAFÍA}

Agamben, G. 2004: Estado de excepción. Homo Sacer II, I. Valencia, Pre-Textos.

Angelis, M. De. 2004: "Separating the Doing and the Deed: Capital and the Continuous Character of Enclosures", Historical Materialism, 12, 2, 56-87. https://doi.org/10.1163/1569206041551609

Arruda, G. 2006: "Historia de ríos: ¿Historia ambiental?", Signos Históricos, 8, 16, 16-44.

Barney, K. 2009: "Laos and the Making of a 'Relational' Resource Frontier", The Geographical Journal, 175, 2, 146-159. https://doi. org/10.1171/j.7475-4959.2009.00323.x

Barona, G., Gómez, A. y Domínguez, C. 1998: "El proceso de construcción territorial de la Orinoquia colombiana en el siglo XIX", en Fajardo Montaña, D. y Urbina, F. (coords): Colombia Orinoco. Bogotá, Fondo FEN, 203-219.

Blackbourn, D. 2008: "'Time is a Violent Torrent': Constructing and Reconstructing Rivers in Modern German History", en Mauch, C. y Zeller, T. (Coords.), Rivers in History. Perspectives on Waterways in Europe and North America. Pittsburgh, University of Pittsburgh Press, 11-25.

Bonnet, J. 1884: Comercio Oriental por el río Meta. Bogotá, Imprenta de La Luz.

CyM. Cal Mayor y Asociados. 1999: Estudio de demanda del río Meta (Informe Final). Bogotá, Ministerio de Transporte. Sin publicar.

Calle, L. 2016: La insaciable búsqueda de El Dorado: procesos hegemónicos y dispositivos de dominación en un pueblo sikuani de la Orinoquia colombiana, tesis doctoral, Universidad Complutense, Madrid.

Cassani, J. 1741: Historia de la Provincia de la Compañía de Jesús del Nuevo Reyno de Granada en la América. Madrid, Imprenta y librería de Manuel Fernández.

Castro, G. 2000: "La crisis ambiental y las tareas de la historia en América Latina", Papeles de población, 6, 24, 37-60. http://dx.doi. org/10.22201/cela.24484946e.1994.2.49681

Coates, P. 2013: A Story of Six Rivers. History, Culture and Ecology. Londres, Reaktion Books.

Cobos, M. T. 1966: "El llanero y la colonización de Oriente", Boletín Cultural y Bibliográfico, 9, 1, 74-78.

Colmenares, G. 1984: "Los jesuitas: modelo de empresarios coloniales", Boletín Cultural y Bibliográfico, 21, 2, 42-53.

Das, V. y Poole, D. 2004: "State and Its Margins. Comparative Ethnographies", en Das, V. y Poole, D. (coords.), Anthropology in the Margins of the State. Nueva Dehli, Oxford University Press, 3-33.

Departamento Nacional de Planeación. 2014: Documento CONPES 3797: Politica para el desarrollo integral de la Orinoquia: Altillanura Fase I. Bogotá, Departamento Nacional de Planeación.

Durán, A. 2012: "Herencias moderno-coloniales en actuales discursos sobre la Orinoquia colombiana", EURE, 38, 115, 195-217. http://dx.doi.org/10.4067/S0250-71612012000300009

\footnotetext{
95. Majumdar, 2016.
} 
Franco, R. 1997: Historia de Orocué. Bogotá, Kelt Colombia S. A. y Ecopetrol.

Gallini, S. 2009: "Historia, ambiente, política: el camino de la historia ambiental en América Latina", Nómadas, 30, 92-102.

García, 0. 2009: "Carimagua: La investigación y el desarrollo en ecosistemas de baja fertilidad", Revista Colombiana de Ciencias Pecuarias, 22, 1, 74-78.

Gómez, A. J. 1991: Indios, colonos y conflictos: una historia regional de los Llanos Orientales 1870-1970. Bogotá, Siglo XXI Editores, Pontificia Universidad Javeriana.

González, L. M. 2015: "El papel de las crónicas misionales coloniales en la configuración de los Llanos Orientales de Colombia y en la producción social de las diferencias", Historia y Sociedad, 29, 17-42. http://dx.doi.org/10.15446/hys.n29.50412.

Gumilla, J. 1944: El Orinoco ilustrado: historia natural, civil y geográfca de este gran río. Bogotá, Ministerio de Educación de Colombia.

Harley, J. B. 1989: "Deconstructing the map", Cartographica: The International Journal for Geographic Information and Geovisualization, 26, 2, 1-20. https://doi.org/10.3138/E635-78271757-9T53

Hernández, N. 2009: "Puerto Carreño: A orillas del Orinoco", Credencial Historia, 229.

IIRSA. 2008: Agenda de implementación consensuada 2005-2010. Informe Junio de 2008. Bogotá, IIRSA.

Junguito, R. 2011: "Transportes Fluviales y Desarrollo Empresarial en Colombia: La Empresa El Libertador de Navegación a Vapor por el Río Meta, 1892-99", Anuario CEEED, 3 3, 45-84.

Langebaek, C. y Morales, J. 2000: Por los caminos del Piedemonte : una historia de las comunicaciones entre los Andes Orientales y los Llanos, siglos XVI a XIX. Bogotá, Universidad de los Andes.

Lugari, P. 1986: "Centro Las Gaviotas. El futuro en las manos", en Torres Acevedo, C. (coords.), Los Llanos de Colombia. Bogotá, Colombia Arco, 187-189.

Majumdar, A. 2016: "The Colonial State and Resource Frontiers: Tracing the Politics of Appropriating Rubber in the Northeastern Frontier of British India, 1810-84", Indian Historical Review, 43, 1, 25-41. https://doi.org/10.1177/0376983616628383.

Mauch, C. y Zeller, T. 2008: "Introduction", en Mauch, C. y Zeller, T (cords.): Rivers in History: Perspectives on Waterways in Europe and North America. Pittsburgh, University of Pittsburgh Press, 1-10.

Mejía, M. 2004: Aventureros, Cronistas y Científicos en la Orinoquia. Bogotá, Universidad Nacional de Colombia.

Melendro, M. 1978: Compañía Nacional de Navegación NAVENAL: fundación y desarrollo 1945-1972. Bogotá, Imprenta Nacional.

Ministerio de Agricultura y Desarrollo Rural. 2004: El Renacimiento de la Orinoquia Alta de Colombia: Un megaproyecto para el mundo. Bogotá, Ministerio de Agricultura y Desarrollo Rural.

Morey, N. K. C. 1975: Ethnohistory of the Colombian and Venezuelan Ilanos, tesis doctoral, University of Utah, Utah.
Núñez, A., Aliste, E. y Bello, A. 2014: "Patagonia-Aysén en la construcción del imaginario geográfico de la nación", Iztapalapa Revista de Ciencias Sociales y Humanidades, 76, 165-188. http:// dx.doi.org/10.28928/ri/762014/aot2/nunezgonzaleza/alistealumnae/bellomaldonadoa

Pacheco, R. 1987: Estudio Navegabilidad en el Río Meta. Bogotá, Colombia, Ministerio de Obras Públicas y Transporte. Sin publicar.

Rausch, J. 1994: Una frontera de la sabana tropical: los Llanos de Colombia, 1537-1831. Bogotá, Banco de la República.

Rausch, J. 1999: La frontera de los Llanos en la historia de Colombia. Bogotá, Banco de la República y El Áncora Editores.

Rausch, J. 2009: "Petroleum and the Transformation of the Llanos Frontier in Colombia: 1980 to the Present", The Latin Americanist, 53, 113-136. https://onlinelibrary.wiley.com/doi/abs/10.1111/ j.1557-203X.2009.01011.x

Rivero, Padre J. 1956: Historia de las Misiones de los Llanos de Casanare y los ríos Orinoco y Meta. Bogotá, Presidencia de la República.

Romero, M. E. 1993: Geografía humana de Colombia. Región de la Orinoquia. Tomo III, vol. 1. Bogotá, Instituto Colombiano de Cultura Hispánica.

Romero-Ruiz, M. H., Flantua, S. G. A., Tansey, K. y Berrio J. C. 2012: "Landscape transformations in savannas of northern South America: Land use/cover changes since 1987 in the Llanos Orientales of Colombia", Applied Geography 32, 2, 766-776. https://doi.org/10.1016/j.apgeog.2011.08.010

Rueda, J. E. 1989: "El complejo económico-administrativo de las antiguas haciendas jesuíticas del Casanare", Boletín Cultural y Bibliográfico, 26, 20, 3-15.

Sánchez, L. F. 2003: Caracterización de los grupos humanos rurales de la cuenca hidrográfica del Orinoco en Colombia. Informe Final. Bogotá, Instituto de Investigaciones Biológicas Alexander von Humboldt.

Santos, J. M. 2016: "Palabras del Presidente Juan Manuel Santos durante la sanción de la Ley de Zidres", el 29 de enero de 2016. http://es.presidencia.gov.co/discursos/Palabras-delPresidente-Juan-Manuel-Santos-durante-la-sancion-de-la-Leyde-Zidres.

Sedrez, L. 2002: "Historia ambiental de América Latina: orígenes, principales interrogantes y lagunas", en Palacio, G. y Ulloa, A. (coords.), Repensando la naturaleza. Encuentros y desencuentros disciplinarios en torno a lo ambiental. Bogotá, Universidad Nacional de Colombia-Sede Leticia, Instituto Amazónico de Investigaciones IMANI, Instituto Colombiano de Antropología e Historia, Colciencias, 99-112.

Serje, M. 2005: El revés de la nación: Territorios salvajes, fronteras y tierras de nadie. Bogotá, Universidad de Los Andes.

Serje, M. 2013: "El mito de la ausencia del Estado: la incorporación económica de las 'zonas de frontera' en Colombia", Cahiers des Amériques latines, 71, 95-117. https://doi.org/10.4000/cal.2679. 
Serje, M. 2017: "Fronteras y periferias en la historia del capitalismo: el caso de América Latina", Revista de geografía Norte Grande, 66, 33-48. https://doi.org/10.4067/s0718-34022017000100003

Silva, G. 2009: Champanes, vapores y remolcadores. Historia de la navegación y la ingeniería fluvial colombiana. Bogotá, Academia Colombiana de Historia de la Ingeniería y las Obras Públicas.

Tovar, H. 2010: Relaciones y Visitas a los Andes. S. XVI. Región de los Llanos. Bogotá, Universidad de Los Andes.

Tsing, A. L. 2005: Friction. An Ethnography of Global Connection. New Jersey, Princeton University Press. https://doi. org/10.1515/9781400830596
Viloria, J. 2009: Geografía económica de la Orinoquia. Cartagena de Indias, Banco de la República.

White, R. 1995: The Organic Machine: the remaking of the Columbia River. New York, Hill and Wang.

Worster, D. 1985: Rivers of Empire. Water, Aridity and the Growth of the American West. Nueva York, Pantheon Books.

Worster, D. 1994: "Thinking like a river", en The Wealth of Nature. Environmental History and the Ecological Imagination. New York y Oxford, Oxford University Press, 123-141. https://doi. org/10.1093/oso/9780195092646.003.0013 